\title{
Efeito da cadência de pedalada sobre a relação entre o limiar anaeróbio e máxima fase estável de lactato em indivíduos ativos do sexo masculino
}

\author{
VDA Ruas \\ TR Figueira \\ F Caputo \\ DF Barbeitos \\ BS Denadai
}

https://doi.org/10.5628/rpcd.06.01.15

\author{
Universidade Estadual Paulista \\ Laboratório de Avaliação da Performance \\ Rio Claro - SP \\ Brasil
}

\section{RESUMO}

O objetivo deste estudo foi analisar a influência da cadência de pedalada na validade do limiar anaeróbio (LAn) em predizer a carga correspondente à máxima fase estável de lactato $\left(\mathrm{MLSS}_{\text {carga }}\right)$, durante o exercício realizado no cicloergômetro. Vinte e oito indivíduos, fisicamente ativos, do sexo masculino $(21,7 \pm 3,5$ anos, $72,7 \pm 10,1 \mathrm{~kg}, 177,0 \pm 4,5 \mathrm{~cm})$ realizaram em uma bicicleta de frenagem mecânica um teste incremental máximo, para determinar o LAn e de 2 a 4 testes de carga constante, para determinar a MLSS carga. Os testes foram realizados nas cadências de 50,60, 70 e $100 \mathrm{rpm}$. O LAn foi determinado como sendo a carga correspondente a $3,5 \mathrm{mM}$ de lactato

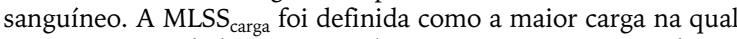
a concentração de lactato sanguíneo não aumentou mais do que $1,0 \mathrm{mM}$ entre o $10^{\circ}$ e o $30^{\circ}$ minuto do teste de carga constante. Não houve diferença significante entre a MLSS $_{\text {carga }}(50$ $\mathrm{rpm}=187,1 \pm 26,7 ; 60 \mathrm{rpm}=182,8 \pm 31,0 ; 70 \mathrm{rpm}=180,2$ $+24,5$ e; $100 \mathrm{rpm}=154,5+24,8$ Watts $)$ e o LAn $(50 \mathrm{rpm}=$ $189,8 \pm 31,5 ; 60 \mathrm{rpm}=175,2 \pm 37,8 ; 70 \mathrm{rpm}=187,2 \pm 28,0$ e; $100 \mathrm{rpm}=142,9 \pm 23,9$ Watts) em nenhuma das cadências analisadas. Com exceção da cadência de $100 \mathrm{rpm}(\mathrm{r}=0,59 ; \mathrm{p}$ $>0,05)$, o LAn foi significantemente correlacionado com a MLSS $_{\text {carga }}(50 \mathrm{rpm}-\mathrm{r}=0,80 ; 60 \mathrm{rpm}-\mathrm{r}=0,96 ; 70 \mathrm{rpm}-\mathrm{r}=$ $0,81)$. Pode concluir-se que, nas cadências de pedalada habitualmente utilizadas (50-70 rpm) em testes incrementais para avaliação de indivíduos sedentários, o LAn apresenta uma boa validade em predizer a MLSS $_{\text {carga }}$.

Palavras-chave: capacidade aeróbia, cicloergômetro, freqüência de pedalada.

\section{ABSTRACT}

Effects of Pedaling Cadence on the Relationship Between Anaerobic Threshold and Maximal Lactate Steady State in Active Male Individuals

The aim of the present study was to analyse the influence of pedaling cadence on the validity of anaerobic threshold (AT) to estimate the exercise workload corresponding to the maximal lactate steady state (MLSS $\left._{\text {workload }}\right)$ during cycle ergometer. Twenty-eight active male $(21.7+3.5 \mathrm{yr}, 72.7+10.1 \mathrm{~kg}, 177.0+4.5 \mathrm{~cm})$ performed one incremental maximal-load test to determine AT and two to four constant submaximal load tests on a mechanically braked cycle ergometer

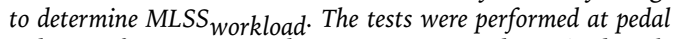
cadences of 50,60, 70 and $100 \mathrm{rpm}$. AT was determined as the workload corresponding to $3.5 \mathrm{mM}$ of blood lactate. The MLSS workload $_{\text {. }}$ was defined as the highest workload at which blood lactate concentration did not increase by more than $1.0 \mathrm{mM}$ between minutes 10 and 30 of the constant workload. There was no significant difference between MLSS $_{\text {workload }}(50 \mathrm{rpm}=187.1 \pm 26.7 ; 60 \mathrm{rpm}=182.8$ $+31.0 ; 70 \mathrm{rpm}=180.2 \pm 24.5$ and; $100 \mathrm{rpm}=154.5+24.8$ Watts $)$ and $A T(50 \mathrm{rpm}=189.8 \pm 31.5 ; 60 \mathrm{rpm}=175.2 \pm 37.8$; $70 \mathrm{rpm}=187.2 \pm 28.0$ and; $100 \mathrm{rpm}=142.9+23.9$ Watts . With exception of cadence at $100 \mathrm{rpm}(r=0.59 ; p>0.05)$, AT was significantly correlated with MLSS workload $_{\text {( }} 50 \mathrm{rpm}-r=0.80 ; 60$ $r p m-r=0.96 ; 70 \mathrm{rpm}-r=0.81)$. We conclude that at cadences more frequently performed in incremental tests $(50-70 \mathrm{rpm})$, AT pre-

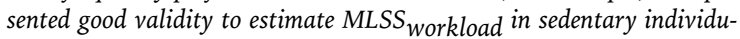
als.

Key Words: aerobic capacity, cycle ergometer, pedaling frequency. 


\section{INTRODUÇÃO}

A máxima fase estável de lactato (MLSS) é a maior concentração de lactato sanguíneo ([Lac $]_{\text {sang }}$ ) que pode ser mantida em fase estável, durante o exercício prolongado de carga constante (3). A MLSS tem sido utilizada para identificar, de modo individualizado, a concentração de lactato e carga constante correspondente (MLSS carga $_{\text {, }}$, acima das quais a taxa de liberação de lactato para o sangue excede sua taxa de remoção deste compartimento. Entre as principais aplicações da identificação da MLSS $_{\text {carga }}$ temos a avaliação dos efeitos de um programa de treinamento aeróbio, a prescrição da intensidade deste treinamento e a categorização dos diferentes domínios da intensidade do exercício (i.e.: moderado, pesado ou severo) (6). Entretanto, a determinação da MLSS carga $_{\text {a }}$ requer que o indivíduo realize de 4 a 5 testes de carga constante, preferencialmente em diferentes dias, com aproximadamente 30 min de duração. Este procedimento aumenta os custos operacionais do laboratório, e pode, também, interferir na rotina de treinamento do atleta.

Em função disto, alguns estudos têm tentado identificar indiretamente a MLSS $_{\text {carga }}$, durante um único exercício incremental. Heck et al. (10) verificaram em um grupo heterogêneo (corredores de endurance e indivíduos ativos) que a carga obtida durante um teste incremental correspondente a $4 \mathrm{mM}$ (LAn) é válida para determinar indiretamente a MLSS durante a corrida. Mais recentemente, Denadai et al. (7) verificaram que o LAn é válido para estimar a MLSS $_{\text {carga }}$ em cicloergômetro, independentemente do estado de treinamento aeróbio dos indivíduos. Muitos estudos têm verificado que a cadência de pedalada altera a resposta metabólica ao exercício, tanto para a mesma carga absoluta (Watts) ou relativa de esforço $\left(\% \mathrm{VO}_{2} \max \right)(5)$. O $\mathrm{VO}_{2}$ e a $[\mathrm{Lac}]_{\text {sang }}$ são mais elevadas em cadências maiores (5). O recrutamento das fibras musculares e o fluxo sanguíneo intra-muscular também são dependentes da cadência de pedalada $(1,13)$. As cadências mais baixas $(50-60 \mathrm{rpm})$ parecem recrutar mais as fibras do tipo II, enquanto as cadências mais altas (90-100 $\mathrm{rpm}$ ) parecem aumentar o fluxo sanguíneo, para uma mesma carga absoluta de exercício $(1,13)$. Confirmando estas influências, Woolford et al. (14) verificaram que o limiar anaeróbio individual (segundo ponto de inflexão da curva lactato $v s$. intensidade) é dependente da cadência de pedalada. Deste modo, é possível hipotetizar que a cadência de pedalada pode influenciar a validade do LAn para

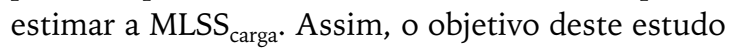
foi analisar a influência da cadência de pedalada sobre a validade do LAn em predizer a MLSS $_{\text {carga }}$ durante o exercício realizado no cicloergômetro.

\section{MATERIAL E MÉTODOS \\ Sujeitos}

Participaram voluntariamente neste estudo 28 sujeitos activos, do sexo masculino $(21,7 \pm 3,5$ anos, 72,7 $\pm 10,1 \mathrm{~kg}, 177,0 \pm 4,5 \mathrm{~cm}$ ), não envolvidos em qualquer programa de treino físico. Os sujeitos foram informados sobre os procedimentos do experimento e suas implicações, tendo assinado um termo de consentimento para a participação no estudo. O protocolo foi aprovado pelo Comitê de Ética em Pesquisa da instituição onde o experimento foi realizado.

\section{Procedimentos experimentais}

Cada voluntário realizou um teste incremental onde foi determinado o LAn, e 2 a 4 testes de carga constante para determinar a MLSS carga $_{\text {em uma bicicleta }}$ de frenagem mecânica (Monark, Brasil). Cada um destes testes foi realizado com a rotação do pedal mantida constante a 50, 60, 70 ou $100 \mathrm{rpm}$. O intervalo entre os testes foi de no mínimo 48 horas, com o protocolo todo durando de 10-15 dias. Os sujeitos não realizaram qualquer esforço intenso nas 48 horas que antecederam cada teste. Em relação a cada voluntário, os testes foram realizados no mesmo local e horário do dia ( \pm 2 horas).

\section{Teste incremental}

Os voluntários foram submetidos a um teste contínuo e incremental, com carga inicial entre 70 e 100 $\mathrm{W}$ e incrementos de $25-35 \mathrm{~W}$ a cada três minutos, até à exaustão voluntária. A freqüência cardíaca (FC) foi anotada no final de cada patamar de carga e as amostras de sangue foram coletadas nos 20 segundos finais de cada estágio. O LAn foi determinado por interpolação linear, utilizando a concentração fixa de 3,5 mM de lactato sanguíneo (10). Foi utilizada esta concentração e não $4 \mathrm{mM}$, em função da duração dos estágios (3 min) do teste incremental. 


\section{Testes de carga constante}

Primeiramente, os sujeitos foram submetidos à intensidade do LAn por trinta minutos ou até a exaustão. Se, durante o primeiro teste de carga constante, observou-se fase-estável ou decréscimo da

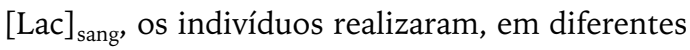
dias, testes de carga constante em intensidades $3-7 \%$ superiores às do teste anterior. Quando, no primeiro teste de carga constante, a [Lac $]_{\text {sang }}$ não apresentou fase-estável ou os indivíduos não concluíram os 30 min de exercício, foram realizados, em diferentes dias, testes de carga constante em intensidades 3-7\% inferiores à do teste anterior. Nestes testes foram coletadas a FC e amostras de sangue do lóbulo da orelha, a cada 5 minutos, para análise do lactato sanguíneo. A MLSS $_{\text {carga }}$ foi definida como sendo a maior carga na qual a [Lac $]_{\text {sang }}$ não aumentou mais do que $1,0 \mathrm{mM}$ entre o $10^{\circ}$ e o $30^{\circ}$ minuto do teste de carga constante (10). A MLSS foi calculada como a média da [Lac $]_{\text {sang }}$ obtida no $10^{\circ}$ e no $30^{\circ}$ minuto da MLSS $_{\text {carga }}(7)$.

\section{Determinação do lactato sanguíneo} e da freqüência cardíaca

Foram coletados $25 \mu \mathrm{l}$ de sangue arterializado do lóbulo da orelha, sem hiperemia, para a determinação do lactato sangüíneo. O sangue foi imediata- mente transferido para microtubulos de polietileno com tampa tipo Eppendorff de 1,5 $\mu$ l, contendo 50 $\mathrm{ml}$ de $\mathrm{NaF} 1 \%$ e este foi armazenado em gelo. A análise do lactato foi realizada através de um analisador eletroquímico (YSL 2300 STAT). A FC foi monitorada através de um frequencímetro (Polar $\mathrm{X}$ - Trainer plus).

\section{Análise estatística}

Os dados estão expressos como média \pm DP. Dentro de cada cadência de pedalada, os valores de MLSS $_{\text {carga }}$ e LAn foram comparados pelo teste $t$ pareado. $\mathrm{O}$ teste de correlação de Pearson foi utilizado para analisar a relação entre MLSS $_{\text {carga }}$ e LAn. Em adição, calculou-se também o nível de concordância entre MLSS $_{\text {carga }}$ e LAn (2). Em todos os testes o nível de significância foi mantido em $5 \%$.

\section{RESULTADOS}

Os valores da MLSS, MLSS $_{\text {carga }}$ e LAn obtidos em cada cadência estão expressos na Tabela 1. Não houve diferença significante entre a MLSS $_{\text {carga }}$ e o LAn em nenhuma das cadências analisadas. Com exceção da cadência de $100 \mathrm{rpm}(\mathrm{r}=0,59$; $\mathrm{p}>$ $0,05)$, o LAn foi significantemente correlacionado com a MLSS $_{\text {carga }}(50 \mathrm{rpm}-\mathrm{r}=0,80 ; 60 \mathrm{rpm}-\mathrm{r}=$ 0,96; $70 \mathrm{rpm}-\mathrm{r}=0,81)$.

\begin{tabular}{l|c|c|c|c|c}
\hline & MLSS $_{\text {carga }}(W)$ & $\begin{array}{c}\text { LAn } \\
(W)\end{array}$ & $\begin{array}{c}\text { FC-MLSS } \\
\text { carga }\end{array}$ & $\begin{array}{c}\text { FC- LAn } \\
\text { (bpm) }\end{array}$ & $\begin{array}{c}\text { MLSS } \\
\text { (bm) }\end{array}$ \\
\hline $50 \mathrm{rpm}$ & 187,1 & 189,8 & 163,1 & 154,2 & 5,2 \\
$(\mathrm{~N}=11)$ & 26,7 & 31,5 & 11,5 & 5,2 & 2,5 \\
\hline $60 \mathrm{rpm}$ & 182,8 & 175,2 & 163,2 & 154,3 & 4,8 \\
$(\mathrm{~N}=7)$ & 31,0 & 37,8 & 10,1 & 10,0 & 1,3 \\
\hline $70 \mathrm{rpm}$ & 180,2 & 187,2 & 163,0 & 160,6 & 4,9 \\
$(\mathrm{~N}=10)$ & 24,5 & 28,0 & 15,8 & 14,6 & 1,7 \\
\hline $100 \mathrm{rpm}$ & 154,5 & 142,9 & 161,0 & 153,9 & 5,0 \\
$(\mathrm{~N}=11)$ & 24,8 & 23,9 & 8,8 & 7,5 & 1,1 \\
\hline
\end{tabular}



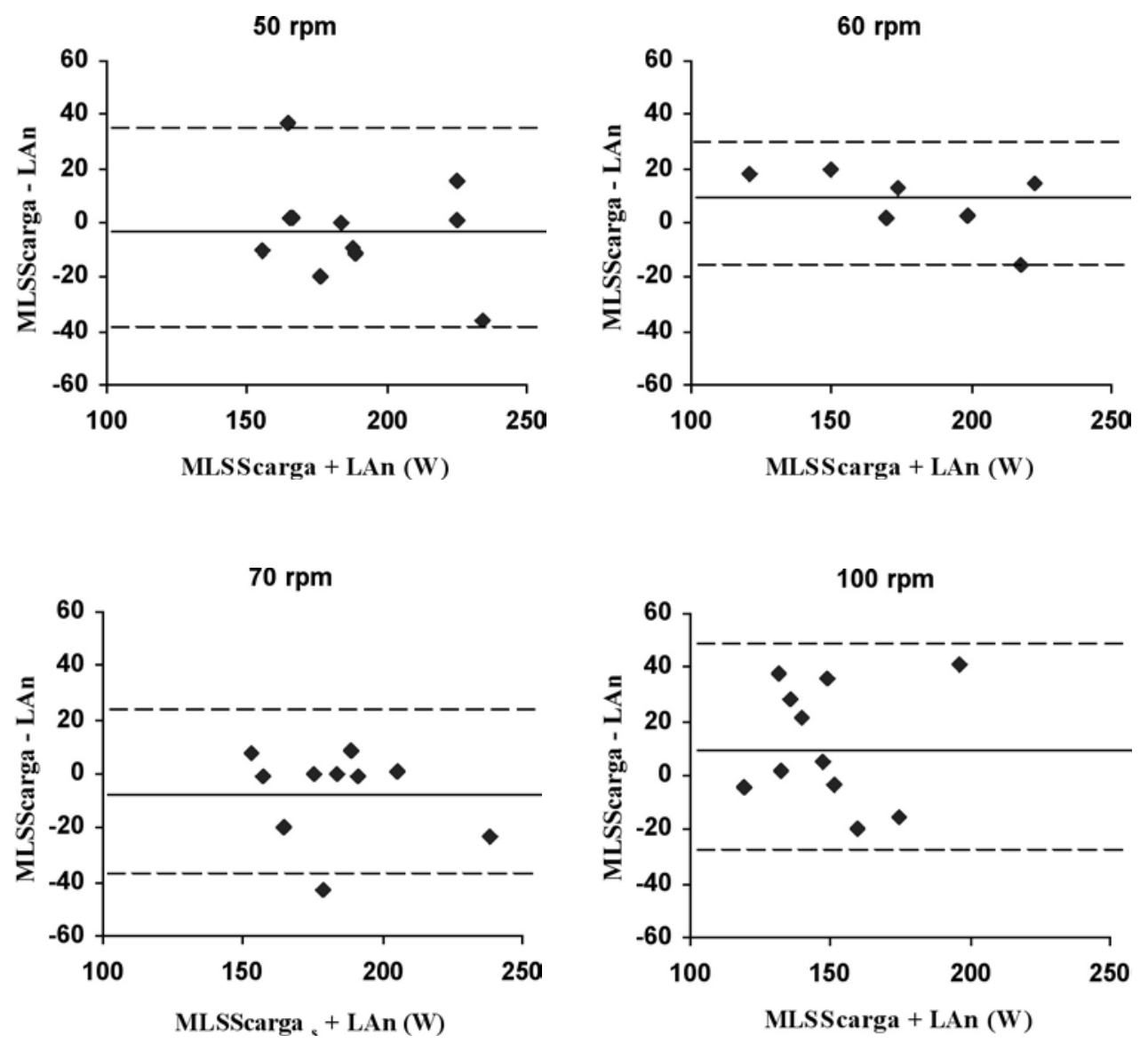

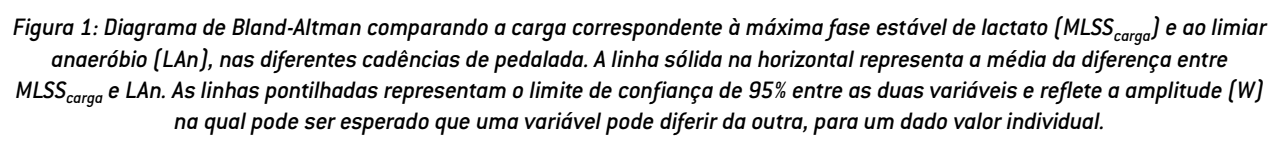

Na Figura 1 estão apresentados os limites de concordância entre o LAn e a MLSS carga $_{\text {para cada uma das }}$ cadências analisadas. Os limites de concordância entre o LAn e a MLSS $_{\text {carga }}$ verificados em $50 \mathrm{rpm}$ [$2,7(18,0) \mathrm{W})], 60 \mathrm{rpm}[7,5(12) \mathrm{W}]$ e $70 \mathrm{rpm}[-7,0$ (16) W], mostram uma baixa variabilidade intraindividual, sugerindo uma boa validade do LAn para

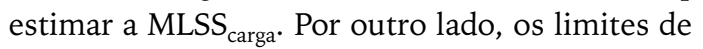
concordância encontrados a 100 rpm [11,6 (21) W], sugerem uma menor validade do LAn para estimar a MLSS $_{\text {carga }}$.

\section{DISCUSSÃO}

Para o nosso conhecimento, este foi o primeiro estudo que analisou a influência da cadência de pedalada sobre a validade do LAn em predizer a MLSS $_{\text {carga }}$ durante o exercício realizado no cicloergômetro. Nosso principal resultado foi que a relação e a con-

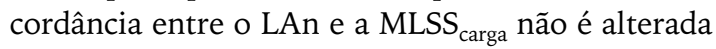
nas cadências de pedalada habitualmente utilizadas (50-70 rpm) em testes incrementais para avaliação de indivíduos sedentários. Muitos estudos têm mostrado que a $[\mathrm{Lac}]_{\text {sang }}$ aumenta em função do incre- 
mento da cadência, para uma dada carga absoluta (watts) ou relativa $\left(\% \mathrm{VO}_{2 \max }\right)$ de exercício $(1,5)$. No entanto, os mecanismos responsáveis por tais alterações ainda não são completamente conhecidos. A cadência de pedalada parece alterar o padrão de recrutamento das unidades motoras (1).

Informações obtidas através da taxa de depleção de glicogênio muscular, sugerem maior recrutamento das fibras tipo II a $50 \mathrm{rpm}$, em relação a $100 \mathrm{rpm}$ (1). Entretanto, cadências mais baixas determinam valores menores de [Lac $]_{\text {sang }}$ do que cadências mais altas, para uma dada carga absoluta (14). Esta relação entre recrutamento das fibras musculares e $[\mathrm{Lac}]_{\text {sang }}$ pode parecer paradoxal. Entretanto, a cadência de pedalada parece influenciar a resposta de lactato, modificando a demanda energética (eficiência mecânica), que é maior nas cadências mais altas. A relação entre $\mathrm{VO}_{2}$ e [Lac $]_{\text {sang }}$ é independente da cadência de pedalada, com ambos aumentando em função do incremento da cadência (5), indicando que $[\mathrm{Lac}]_{\text {sang }}$ é fortemente dependente do gasto energético. Chavarren e Calbet (5) verificaram, também, que a FC para um dado $\mathrm{VO}_{2}$, durante o exercício de carga constante, é independente da cadência empregada. Além disso, Denadai et al. (8) verificaram que a relação entre $\mathrm{FC} \mathrm{e}[\mathrm{Lac}]_{\text {sang }}$ durante o exercício incremental ou de carga constante $\left(\right.$ MLSS $_{\text {carga }}$ ) é independente da cadência (50 x 100 $\mathrm{rpm}$ ). Estes resultados também foram observados no presente estudo, pois a FC correspondente ao LAn e a MLSS $_{\text {carga }}$ são bem semelhantes entre as cadências. O uso de uma determinada concentração fixa de lactato sanguíneo (3,5 ou 4,0 mM para estágios de 3 e 5 min, respectivamente), obtida durante o teste incremental (LAn) para determinar indiretamente a MLSS $_{\text {carga }}$ (10), tem sido criticado por diferentes autores $(12,15)$. Entretanto, muito poucos laboratórios têm comparado o LAn com a MLSS $_{\text {carga }}$ determinada de modo direto e independente, particularmente em cicloergômetro. Em um estudo recente, Denadai et al. (7) verificaram que a validade do LAn para estimar a MLSS $_{\text {carga }}$ em cicloergômetro é independente do estado de treinamento aeróbio (sedentários $\mathrm{x}$ treinados). Os resultados do presente estudo ampliam e confirmam parte dos dados citados anteriormente. Deve-se salientar que a cadência poderia ter influenciado a validade do LAn, principalmente quando se considera a duração do estágio no exercício incremental (3 min). Cadências de pedaladas mais elevadas favorecem o fluxo intramuscular (13), o que poderia acelerar o transporte de lactato entre o compartimento de produção (musculatura ativa) e o compartimento de análise (sangue arterial) (9). Isto, em potencial, elevaria precocemente a concentração de lactato para um valor mais próximo daquele que, em tese $(3,5 \mathrm{mM})$, indicaria a MLSS $_{\text {carga }}$ no teste incremental com estágio de 3 minutos. No entanto, a falta de validade do LAn a $100 \mathrm{rpm}$ foi em função da maior variabilidade e não porque ele subestimou a MLSS $_{\text {carga }}$ determinada na mesma cadência.

Adicionalmente, a MLSS $_{\text {carga }}$ seria menos influenciada por estas alterações, já que sua determinação é realizada durante o exercício de maior duração (30 min) e com carga constante. $\mathrm{O}$ estado de treinamento aeróbio $(4,7)$ e as características histoquímicas (MCT 1 e 4, tipo de fibra e enzimas aeróbias) da fibra muscular (11) parecem não influenciar a MLSS durante o ciclismo. Do mesmo modo, verificamos em nosso estudo que a MLSS parece não ser modificada pela cadência de pedalada, embora a MLSS $_{\text {carga }}$ diminua com aumento da cadência (8).

\section{CONCLUSÃO}

Com base em nossos resultados, podemos concluir que a validade do LAn para a predição da MLSS $_{\text {carga, }}$ nas cadências mais comumente empregadas (50 - 70 rpm), não é influenciada pela cadência de pedalada, durante o ciclismo, em indivíduos ativos. Sugere-se a realização de mais estudos que possam analisar esta influência em outras cadências (p. ex., 80, 90) e/ou em indivíduos com diferentes níveis de treinamento no ciclismo.

\section{CORRESPONDÊNCIA}

Benedito S. Denadai

Laboratório de Avaliação da Performance Humana IB - UNESP

Av. 24 A, 1515 - Bela Vista

13506-900 Rio Claro - SP

BRASIL

bdenadai@rc.unesp.br 


\section{REFERÊNCIAS BIBLIOGRÁFICAS}

1. Ahlquist LE, Basset Jr DR, Sufit R, Nagle FJ, Thomas DP (1992). The effect of pedaling frequency on glycogen depletion rates in type I and type II quadriceps muscle fibers during submaximal cycling exercise. Eur J Appl Physiol 65: 360-364.

2. Bland JM, Altman DG (1986). Statistical methods for assessing agreement between two methods of clinical measurement. Lancet 1: 307-310.

3. Beneke R (2003). Methodological aspects of maximal lactate steady state-implications for performance testing. Eur J Appl Physiol 89: 95-99.

4. Beneke R, Hutler M, Leithauser RM (2000). Maximal lactate steady state independent of performance. Med Sci Sports Exerc 32: 1135-1139.

5. Chavarren J, Calbet J (1999). Cycling efficiency and pedaling frequency in road cyclists. Eur J Appl Physiol 80: 555563.

6. Denadai BS, Caputo F (2003). Efeitos do treinamento sobre a cinética do consumo de oxigênio durante o exercício realizado nos diferentes domínios de intensidade de esforço. Motriz 9: 1-7.

7. Denadai BS, Figueira TR, Favaro ORP, Gonçalves M (2004). Effect of the aerobic capacity on the validity of the anaerobic threshold for determination of the maximal lactate steady state in cycling. Braz J Med Biol Res 37: 15511556.
8. Denadai BS, Ruas VDA, Figueira TR (In press). Efeito da cadência de pedalada sobre as respostas metabólica e cardiovascular durante o exercício incremental e de carga constante em indivíduos ativos. Rev Bras Med Esporte.

9. Gladden L (2000). Muscle as a consumer of lactate. Med Sci Sports Exerc 32: 764-771.

10. Heck H, Mader A, Hess G, Mucke S, Muller R, Hollmann W (1985). Justification of the $4 \mathrm{mmol} / \mathrm{l}$ lactate threshold. Int J Sports Med 6: 117-130.

11. Loekkegaard J, Pedersen PK, Juel C, Sjoegaard G (2001). Individual variations in maximal lactate steady state and their relationship with muscle buffering capacity and lactate transporters. Med Sci Sports Exerc 33: S330.

12. Stegmann H, Kindermann W, Schnabel A (1981). Lactate Kinetics and individual anaerobic threshold. Int J Sports Med 2: 160-165.

13. Takaishi T, Yasuda Y, Ono T, Moritani T (1996). Optimal pedaling rate estimated from neuromuscular fatigue for cyclists. Med Sci Sports Exerc 28: 1492-1497.

14. Woolford S, Withers R, Craig N, Bourdon P, Stanef T, McKenzie I (1999). Effect of pedal cadence on the accumulated oxygen deficit, maximal aerobic power and blood lactate transition thresholds of high-performance junior endurance cyclists. Eur J Appl Physiol 80: 285-291.

15. Van Schuylenbergh R, Eynde BV, Hespel P (2004). Prediction of sprint triathlon performance from laboratory tests. Eur J Appl Physiol 91: 94-99. 